Check for updates

Cite this: RSC Adv., 2018, 8, 39918

Received 23rd August 2018

Accepted 22nd November 2018

DOI: $10.1039 / \mathrm{c} 8 \mathrm{ra07054g}$

rsc.li/rsc-advances

\title{
Nanocellulose/polypyrrole aerogel electrodes with higher conductivity via adding vapor grown nano- carbon fibers as conducting networks for supercapacitor application $\dagger$
}

\author{
Yanping Chen, ${ }^{\text {ab }}$ Shaoyi Lyu, (D *b Shenjie Han, ${ }^{\mathrm{b}}$ Zhilin Chen, ${ }^{\mathrm{b}}$ Wenjun Wang*a \\ and Siqun Wang (iD cb
}

\begin{abstract}
Nanocellulose-based conductive materials have been widely used as supercapacitor electrodes. Herein, electrode materials with higher conductivity were prepared by in situ polymerization of polypyrrole (PPy) on cellulose nanofibrils (CNF) and vapor grown carbon fiber (VGCF) hybrid aerogels. With increase in VGCF content, the conductivities of CNF/VGCF aerogel films and CNF/VGCF/PPy aerogel films increased. The $\mathrm{CNF} / \mathrm{VGCF}_{2} / \mathrm{PPy}$ aerogel films exhibited a maximum value of $11.25 \mathrm{~S} \mathrm{~cm}$, which is beneficial for electron transfer and to reduce interior resistance. In addition, the capacitance of the electrode materials was improved because of synergistic effects between the double-layer capacitance of VGCF and pseudocapacitance of PPy in the CNF/VGCF/PPy aerogels. Therefore, the CNF/VGCF/PPy aerogel electrode showed capacitances of $8.61 \mathrm{~F} \mathrm{~cm}^{-2}$ at $1 \mathrm{mV} \mathrm{s}^{-1}$ (specific area capacitance) and $678.66 \mathrm{~F} \mathrm{~g}^{-1}$ at $1.875 \mathrm{~mA} \mathrm{~cm}{ }^{-2}$ (specific gravimetric capacitance) and retained $91.38 \%$ of its initial capacitance after 2000 cycles. Furthermore, an all-solid-state supercapacitor fabricated by the above electrode materials exhibited maximum energy and power densities of $15.08 \mathrm{~W} \mathrm{~h} \mathrm{Kg}^{-1}$, respectively. These electrochemical properties provide great potential for supercapacitors or other electronic devices with good electrochemical properties.
\end{abstract}

\section{Introduction}

Nanocelluloses have recently attracted significant attention in the field of energy storage due to their light weight, high mechanical strength, environmental friendliness, and biodegradability. In general, nanocelluloses can be classified as bacterial cellulose (BC), cellulose nanofibrils (CNFs), and cellulose nanocrystals (CNCs). ${ }^{1}$ All of these have been extensively exploited to fabricate energy devices by combining them with conducting electroactive materials through various methods, such as physical blending, coating, layer-by-layer assembly, and in situ polymerization. ${ }^{2-6}$ Among these methods, in situ polymerization is a simple method that has been broadly adopted for preparing nanocellulose-based active

aBeijing Engineering Research Center of Cellulose and Its Derivatives, School of Materials Science and Engineering, Beijing Institute of Technology, Beijing 100081, China.E-mail:wangwenjun@bit.edu.cn

${ }^{b}$ Research Institute of Wood Industry, Chinese Academy of Forestry, Beijing 100091, China.E-mail: lvsy@caf.ac.cn

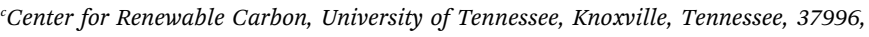
USA

$\dagger$ Electronic supplementary information (ESI) available: [DETAILS]. See DOI: $10.1039 / \mathrm{c} 8 \mathrm{ra} 07054 \mathrm{~g}$ electrode materials with an extra substrate or nanocelluloses as the substrate.

Of the possible conducting electroactive materials, conductive polymers like polyaniline (PANI) and polypyrrole (PPy), which are inexpensive and can be easily synthesized, are widely used in electrode materials because they exhibit high specific mass capacitances and power densities. ${ }^{7-10}$ Nevertheless, due to the insulating nature of CNFs and the limited conductivity of conductive polymers, CNF-based active electrode materials still show low conductivity, preventing the electrode materials from exhibiting higher specific capacitances. In addition, high conductivity is necessary for excellent electrochemical performances when CNF-based active electrode materials are employed as electrodes and current collectors. To solve these problems, efforts have been made by adding a stainless-steel mesh as a current collector, ${ }^{11}$ or by adding highly conductive materials such as metals ${ }^{12-14}$ and carbon fibers. ${ }^{15,16}$ However, the use of an additional current collector or metal increases the weight of the entire electrode and adds huge costs of production, making these methods uneconomical and hard to popularizer. Furthermore, carbon fibers are still too large to be dispersed homogeneously in CNFs.

Using vapor grown carbon fibers (VGCFs), our group adopted fibers with diameters around $150 \mathrm{~nm}$, which allows them to be 
easily dispersed homogeneously in CNFs. Furthermore, VGCFs are cheaper to produce than carbon nanotubes (CNTs) and graphene oxides (GOs). ${ }^{17}$ To the best of our knowledge, although VGCFs have been extensively exploited in Li-ion batteries and fuel cells due to their improved conductivity and cycle stability and reduced internal resistances in electrodes, ${ }^{18-21}$ there have been few reports on the use of VGCFs as electrode materials for supercapacitors.

Herein, we present aerogel electrodes formed by in situ polymerization of PPy on CNF/VGCF aerogel substrates. Due to their unique hollow structures, VGCFs have high specific surface areas and provide more channels for ion transport. In addition, the obtained CNF/VGCF aerogels formed a porous network structure, which was beneficial for absorbing more electrolyte and provided more sites for redox reactions caused by the pseudocapacitance performance of the conductive polymer. Therefore, the electrodes of the all-solid-state supercapacitor showed high specific capacitances of $8.61 \mathrm{~F} \mathrm{~cm}^{-2}$ at $1 \mathrm{mV} \mathrm{s}^{-1}$ (specific area capacitance) and $678.66 \mathrm{~F} \mathrm{~g}^{-1}$ at 1.875 $\mathrm{mA} \mathrm{cm} \mathrm{cm}^{-2}$ (specific gravimetric capacitance) and retained $91.38 \%$ of their initial capacitance after 2000 cycles. Therefore, these electrodes have potential applications in smart and portable supercapacitors and light-weight wearable electric devices.

\section{Experimental section}

\section{Materials}

CNFs with TEMPO-mediated oxidation was provided by Tianjin Haojia Cellulose Co., Ltd. VGCFs were purchased from Beijing Dk Nano technology Co., Ltd. Pyrrole (Py) was purchased from Aladdin and was distilled under reduced pressure before use. Analytical grade tert-butyl alcohol (TBA) was purchased from Beijing Yili Fine Chemicals Co., Ltd. Hydrochloric acid and ferric chloride were purchased from the Beijing Chemical Reagents Co., Ltd. Sodium hypophosphite (SHP) and 1,2,3,4butanetetracarboxylic acid (BTCA) were purchased from SigmaAldrich Co., Ltd.

\section{Preparation of cross-linked CNF/VGCF aerogels}

CNFs mixed with BTCA and SHP were dispersed in deionized water and ultrasonicated using a sonicator (VCX1500, USA) for $30 \mathrm{~min}$. Then, TBA (20 wt\%) and VGCFs were added to the CNF suspension $(0.15 \mathrm{wt} \%)$ with sonication for another $30 \mathrm{~min}$ to disperse them homogeneously. The resulting CNF/VGCF aqueous mixture was transferred to several circular bottles and frozen in a freezer at $-26{ }^{\circ} \mathrm{C}$, followed by freeze-drying using a freeze drier (VirTis Freeze Mobile 25 EL Sentry 2.0, USA). Finally, the freeze-dried aerogel was heated in an oven at $170{ }^{\circ} \mathrm{C}$ for $5 \mathrm{~min}$ to obtain a cross-linked CNF/VGCF aerogel.

Herein, the mass ratios of CNFs (dry wt\%) to VGCFs were 1 / $0.2,1 / 0.5,1 / 1$, and $1 / 2$. Accordingly, the cross-linked CNF/VGCF aerogels were denoted $\mathrm{CNF} / \mathrm{VGCF}_{0.2}, \mathrm{CNF} / \mathrm{VGCF}_{0.5}, \mathrm{CNF} / \mathrm{VGCF}_{1}$, and $\mathrm{CNF} / \mathrm{VGCF}_{2}$ aerogels, respectively. In addition, the weight percentages of BTCA and SHP were constant at $0.045 \%$ and $0.0225 \%$, respectively.

\section{In situ polymerization of CNF/VGCF/PPy aerogel}

CNF/VGCF/PPy aerogels were obtained by in situ polymerization. Firstly, $2 \mathrm{~g}$ of Py was dispersed in $50 \mathrm{~mL}$ of a $0.3 \mathrm{M}$ hydrochloric acid solution. In addition, $10 \mathrm{~g}$ of ferric chloride was dissolved in $50 \mathrm{~mL}$ of a $0.3 \mathrm{M}$ hydrochloric acid solution. The cross-linked CNF/VGCF aerogel was then soaked in the Py dispersion for $30 \mathrm{~min}$ followed by the addition of $0.1 \mathrm{~g} \mathrm{mL^{-1 }}$ ferric chloride solution slowly at $4{ }^{\circ} \mathrm{C}$ for $1 \mathrm{~h}$. A ferric chloride solution was the reaction initiator used to achieve in situ polymerization. After the reaction was complete, the resulting aerogel was washed thoroughly with a $0.3 \mathrm{M}$ hydrochloric acid solution and deionized water in sequence to remove residues. Finally, the washed aerogel was freeze-dried overnight.

\section{Fabrication of all-solid-state symmetric supercapacitor devices}

The CNF/VGCF/PPy aerogel was formed into a thin film under pressure to act as electrodes. Then, the aerogel film was cut to one size and one side was connected to an aluminum sheet using a conductive silver paste, after which the prepared electrode was immersed in the polyvinyl alcohol (PVA) $/ \mathrm{H}_{3} \mathrm{PO}_{4}$ gel electrolyte overnight and dried completely in air. Lastly, two CNF/VGCF/PPy film electrodes, separated by $\mathrm{PVA} / \mathrm{H}_{3} \mathrm{PO}_{4}$ gel electrolyte, were pressed gently to build a sandwich structure for the all-solid-state symmetric supercapacitor devices.

\section{Characterization}

The microstructures and surface morphologies of the crosslinked CNF/VGCF aerogels and CNF/VGCF/PPy aerogels were observed by scanning electron microscopy (SEM, Hitachi S4800, Japan) at $5.0 \mathrm{kV}$ and transmission electron microscopy (TEM, FEI Tecnai G2 F30).

Fourier transform infrared spectroscopy (FT-IR, Nicolet iS10, Thermo Fisher Scientific Inc., USA) and Raman spectroscopy (Renishaw, UK) with $632 \mathrm{~nm}$ laser excitation were employed to observe the vibrational characterizations of the functional groups. X-ray photoelectron spectroscopy (XPS) was conducted on a Thermo ESCALAB 250Xi System (Thermo Electron Scientific Instruments Co. Ltd., USA) with an Al- $\mathrm{K}_{\alpha} \mathrm{X}$-ray source (1486.6 eV). The X-ray diffraction (XRD) was conducted on a Bruker D8 Advance System using $\mathrm{Cu}-\mathrm{K}_{\alpha}$ radiation at $\lambda=$ $1.5406 \AA$.

Brunauer-Emmett-Teller (BET) and Barrett-Joyner-Halenda (BJH) models were applied to analyze the specific surface areas and pore size distributions of the aerogels with an $\mathrm{N}_{2}$ adsorption analyzer (ASAP 2460, Micromeritics Instrument Corp., USA).

Conductivity was measured using an ST 2253 4-point probe resistivity measurement system (Suzhou Jingge Electronic Co., Ltd, China). Electrochemical characterization was performed by a two-electrode test and three-electrode test using a CHI 660E electrochemical workstation (Shanghai Chenhua Instrument Co., LTD, China). In the three-electrode test, cyclic voltammetry (CV) and galvanostatic charge-discharge (GCD) were performed between $0-0.8 \mathrm{~V}$ in $1 \mathrm{M} \mathrm{H}_{2} \mathrm{SO}_{4}$ electrolyte solution with 
a platinum plate electrode as the counter electrode and a saturated calomel electrode (SCE) as the reference electrode. The two-electrode test was employed to perform electrochemical measurements of the prepared supercapacitor devices in a potential window of $0-0.8 \mathrm{~V}$. Electrochemical impedance spectroscopy (EIS) was carried out over the frequency range of 0.001 to $10^{5} \mathrm{~Hz}$.

The areal energy $E\left(\mathrm{~W} \mathrm{~h} \mathrm{~kg}^{-1}\right)$ and the areal power $P\left(\mathrm{~W} \mathrm{~kg}^{-1}\right)$ of the supercapacitor were obtained according to the expressions $^{22,23}$

$$
\begin{gathered}
E=I\left(\int U \mathrm{~d} t\right) /(3.6 m) \\
P=3600 E / \Delta t, \text { respectively. }
\end{gathered}
$$

Where $I(\mathrm{~A}), U(\mathrm{~V}), \Delta t(\mathrm{~s})$, and $m(\mathrm{~g})$, are the current, working area of the electrode, discharge time, and total electrode weight, respectively.

For the three-electrode test, the areal specific capacitance, $C_{\mathrm{s}}$ $\left(\mathrm{F} \mathrm{cm}^{-2}\right)$, and the gravimetric specific capacitance, $C_{\mathrm{g}}\left(\mathrm{F} \mathrm{g}^{-1}\right)$, were calculated as follows:

$$
\begin{gathered}
C_{\mathrm{s}}=\left(\int I \mathrm{~d} U\right) /(v S U) \\
C_{\mathrm{g}}=2 I\left(\int U \mathrm{~d} t\right) /\left(m U^{2}\right)
\end{gathered}
$$

where $v\left(\mathrm{~V} \mathrm{~s}^{-1}\right)$ and $S\left(\mathrm{~cm}^{2}\right)$ are the current and working area of the electrode.

For the two-electrode test:

$$
\begin{gathered}
C_{\mathrm{s}}^{\prime}=4\left(\int I \mathrm{~d} U\right) /(v S U) \\
C_{\mathrm{g}}^{\prime}=8 I\left(\int U \mathrm{~d} t\right) /\left(m U^{2}\right)
\end{gathered}
$$

where $C_{\mathrm{s}}^{\prime}$ is the areal specific capacitances and $C_{\mathrm{g}}^{\prime}$ is the gravimetric specific capacitance of the supercapacitor device. ${ }^{23}$

\section{Results and discussion}

As shown in Fig. 1, the simple preparation process of the $\mathrm{CNF}$ / VGCF and CNF/VGCF/PPy aerogels includes ultrasonication, freeze-drying, and in situ polymerization. CNF/VGCF aerogels were cross-linked using BTCA and SHP to avoid severe deformation and structural damage during in situ polymerization.

As shown in Fig. S3(a), $\dagger$ the VGCFs resemble slender rods with 5-10 um lengths and 100-150 nm diameters. VGCFs also have a hollow tubular structure as shown in Fig. S3(b), $\dagger$ which is beneficial for the storage of electrolyte. It is known that CNF aerogels have a good porous structure, which can be used as a nano-reservoir for electrolyte. To investigate the effect of VGCFs on the structure and performance of composite aerogels, aerogels were prepared with different VGCF contents (the mass ratios of CNFs to VGCFs are $1 / 0.2,1 / 0.5,1 / 1,1 / 2$, respectively). As shown in Fig. S1(a)-(c), $\dagger$ 2(a) and (b), all the CNF/VGCF aerogels formed porous network structures, where fibers of CNFs and VGCFs were interlaced and the surfaces of the fibers were smooth. This can be also observed in the TEM images shown in Fig. 3(a), where CNFs and VGCFs are dispersed homogeneously and interwoven. It is known that carbon fibers have high strengths. Therefore, in our research, VGCFs embedded in CNFs could increase the strength of the composite aerogels, protecting the structure from damage during in situ polymerization or pressing into films. Also, the fibers of the CNFs became slenderer, the interlaced fibers of CNF/VGCF aerogels were more plentiful in number, and the sheet structures formed by the CNFs diminished with increase in VGCF content. The pore sizes increased with increase in VGCF content, which can be also concluded from the values of average pore diameters shown in Table S1. $\dagger$ As shown in Fig. S1(d)-(f), $\dagger$ 2(d) and (e), the surfaces of the fibers in the CNF/VGCF/PPy aerogels with different VGCF contents were rougher compared to those of the CNF/VGCF aerogels. In addition, the fibers were coated with several layers of blobs, which are PPy formed by chemical polymerization. We can see these more clearly in Fig. 3(c) and (d), where there is an obvious porous structure that is unaffected by the adhesion of PPy. This also indicates that the time allotted for in situ polymerization was sufficient for obtaining an adequate content of PPy. The cross-sectional images of the $\mathrm{CNF} / \mathrm{VGCF}_{1}$ and $\mathrm{CNF} / \mathrm{VGCF}_{1} / \mathrm{PPy}$ aerogels after being pressed into films shown in Fig. 2(c) and (f), respectively, show layered structures in which many pores can be seen. It can be also seen that the aerogels retained their porous structures when VGCFs were embedded in the CNFs. The structures of the cross-section of CNF/VGCF/PPy with other VGCF contents were similar to that of the $\mathrm{CNF} / \mathrm{VGCF}_{1} / \mathrm{PPy}$ aerogel film (Fig. S2 $\dagger$ ).

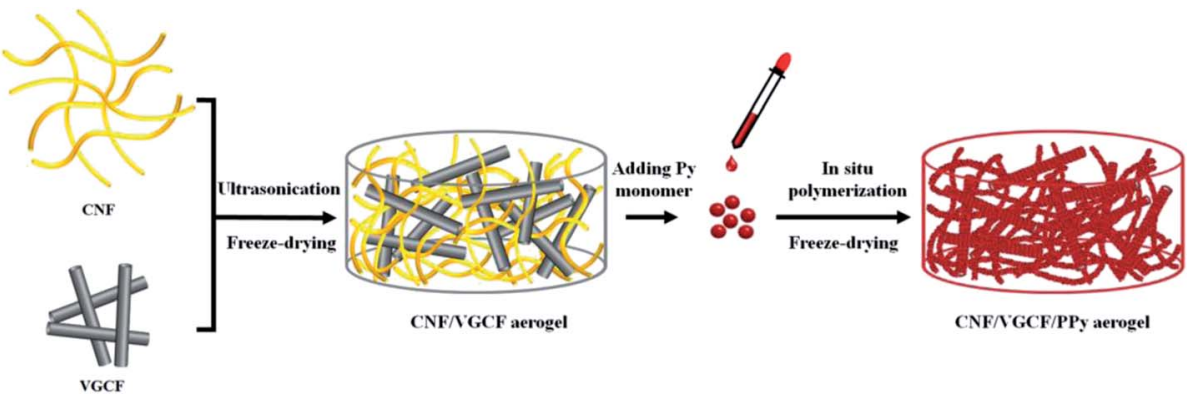

Fig. 1 Schematic of the preparation process of CNF/VGCF/PPy aerogels. 

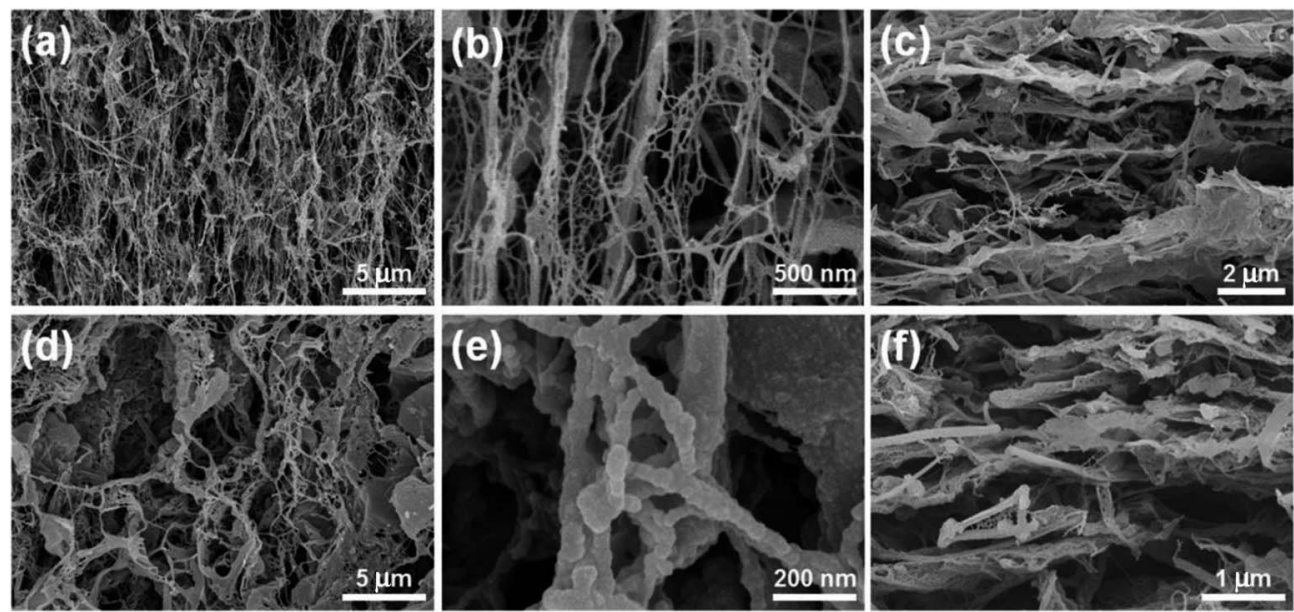

Fig. 2 SEM images of CNF/VGCF 1 aerogel: (a) an internal surface image of the aerogel, (b) a magnified image of the aerogel, and (c) a crosssection image of the aerogel film. SEM images of $\mathrm{CNF}_{\mathrm{NGCF}} / \mathrm{PPy}$ aerogel: (d) an internal surface image of the aerogel, (e) a magnified image of the aerogel, and (f) a cross-sectional image of the aerogel film.

To further analyze the porous structures of the CNF/VGCF aerogels with different VGCF contents, Brunauer-EmmettTeller (BET) and Barrett-Joyner-Halenda (BJH) models were used to calculate pore size distributions and the specific surface areas. Fig. 4(a) shows the typical nitrogen adsorption-desorption isotherms of the CNF/VGCF aerogels with different VGCF contents. The isotherms are between type II and type IV of the IUPAC isotherm classification system..$^{24,25}$ The isotherms increase gently with $P / P^{0}$ for $P / P^{0}<0.1$, indicating the presence of only a few micropores. The adsorption hysteresis loops indicate the existence of mesopores and capillary condensation in the mesopores or macropores. ${ }^{26}$ The curves of the pore size distributions of the CNF/VGCF aerogels with different VGCF contents exhibit sharp peaks at 2-20 nm (Fig. S4(a)†). This indicates that most pore sizes of the aerogels were in the 2$20 \mathrm{~nm}$ range, indicating that a large number of mesopores existed, which act as pathways for ion transfer in general. More details of the porous properties of the CNF/VGCF aerogels with
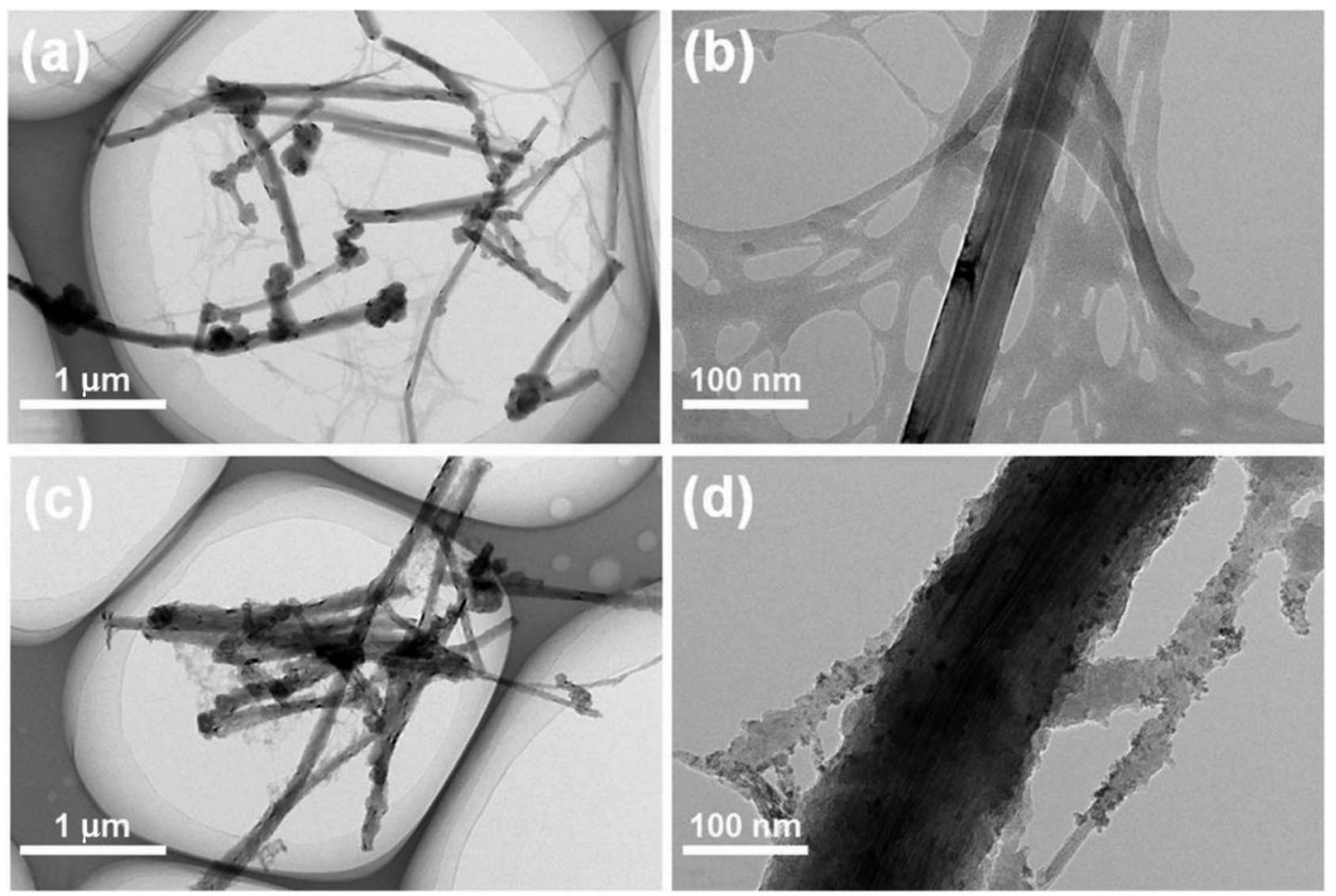

Fig. 3 TEM images of (a) CNF/VGCF 1 aerogel and (c) CNF/NGCF 1 /PPy aerogel. Magnified TEM images of (b) CNF/VGCF 1 aerogel and (d) CNF/ $\mathrm{VGCF}_{1} /$ PPy aerogel. 

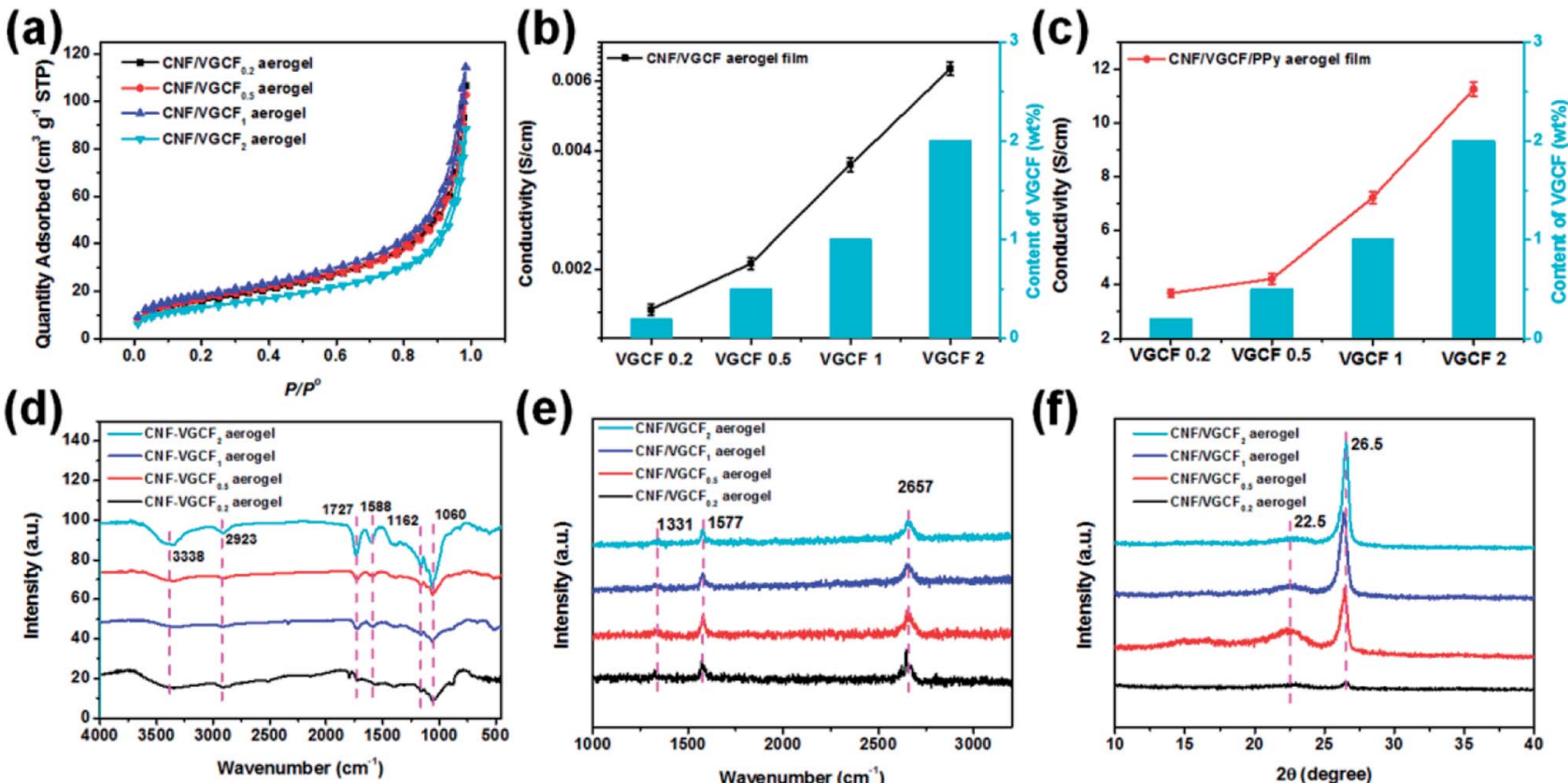

(e)
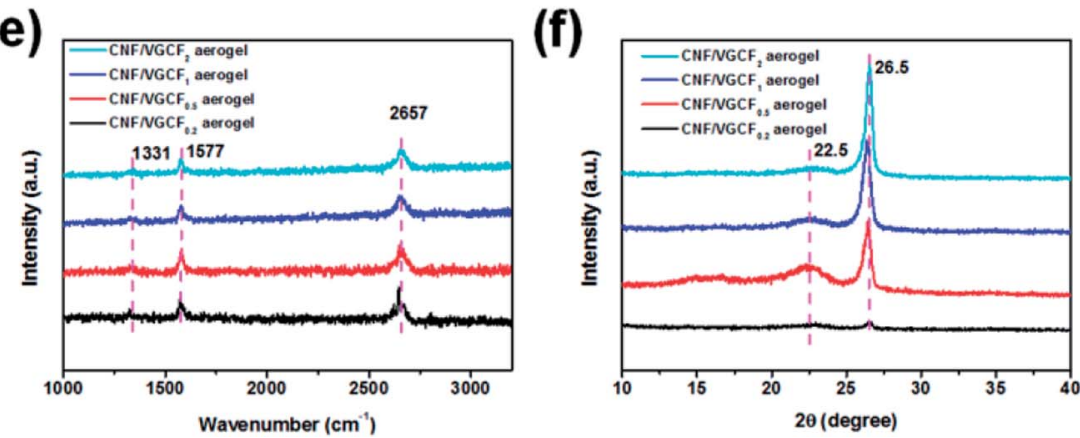

Fig. 4 (a) Typical nitrogen adsorption desorption of CNF/VGCF aerogels with different VGCF contents. Conductivities of (b) CNF/VGCF aerogels and (c) CNF/VGCF/PPy aerogels with different VGCF contents. (d) FTIR spectra, (e) Raman spectra, and (f) XRD spectra of CNF/VGCF aerogels with different VGCF contents.

different VGCF contents are shown in Table S1.† The CNF/ $\mathrm{VGCF}_{1}$ aerogel had a maximum BET specific surface area of $67.18 \mathrm{~m}^{2} \mathrm{~g}^{-1}$, followed by CNF/VGCF $0.5\left(61.51 \mathrm{~m}^{2} \mathrm{~g}^{-1}\right), \mathrm{CNF} /$ VGCF $_{0.2}\left(50.93 \mathrm{~m}^{2} \mathrm{~g}^{-1}\right)$, and CNF/VGCF $2\left(47.81 \mathrm{~m}^{2} \mathrm{~g}^{-1}\right)$ aerogels. Maximum total pore volume of the CNF/VGCF aerogels was also achieved when the ratio of CNFs to VGCFs is $1 / 1$. However, the $\mathrm{CNF} / \mathrm{VGCF}_{2}$ aerogel had the lowest specific surface area and total pore volume of the samples. The values of the average pore diameter increased with the increase in content of VGCFs, which is consistent with the shape variation of the pores observed in the SEM images. For the results above, it can be concluded that the $\mathrm{CNF} / \mathrm{VGCF}_{1}$ aerogel formed a better porous structure and had a relatively high specific surface area. These results also show that the addition of VGCFs indeed affected the specific surface areas, pore sizes, and total pore volumes of the composite aerogels, while the composite aerogels retained their porous structures, and there was not a positive correlation between the structural variation trends and the content of VGCF.

Conductivity is one of the key factors affecting the final electrochemical properties. It has been reported that electrode materials with higher conductivities have a greater capacity for electron-transport. It is known that pure CNFs have poor conductivity. Herein, VGCFs were added to CNF aerogels to increase the conductivity. It can be seen in Fig. 4(b) that the conductivities of the CNF/VGCF aerogels were improved by adding VGCFs and increased with increase in VGCF content. When the ratio of CNFs to VGCFs was $1: 2$, the conductivity reached a maximum value of $6.46 \times 10^{-3} \mathrm{~S} \mathrm{~cm}^{-1}$. Fig. 4(c) shows that the conductivities of the CNF/VGCF/PPy aerogel films increased from $3.69 \mathrm{~S} \mathrm{~cm}^{-1}$ to $11.25 \mathrm{~S} \mathrm{~cm}^{-1}$ with increase in VGCF content. These results are superior to those of PPy/ cellulose nanopapers or composites..$^{5,27-29}$ They are also higher than the Ag/MWCNT/cellulose paper reported previously. ${ }^{30}$ Herein, the CNF/VGCF/PPy aerogel films with relatively high conductivities were expected to show higher specific capacitance, which was verified by electrochemical tests, discussed below.

Fig. 4(d) shows the FTIR spectra of the CNF/VGCF aerogels with different VGCF contents. The band at $3338 \mathrm{~cm}^{-1}$ corresponds to $\mathrm{O}-\mathrm{H}$ stretching vibrations of hydroxyl groups and the band at $2923 \mathrm{~cm}^{-1}$ corresponds to $\mathrm{C}-\mathrm{H}$ stretching vibrations in the CNFs. ${ }^{31}$ The band at $1727 \mathrm{~cm}^{-1}$ corresponds to $\mathrm{C}=\mathrm{O}$ stretching vibrations of hydroxyl groups in the CNFs and the peak at $1588 \mathrm{~cm}^{-1}$ corresponds to $\mathrm{C}=\mathrm{C}$ stretching of the $\mathrm{sp}^{2}$ structure of the VGCFs. ${ }^{17}$ The absorption peaks at 1530, 1382, 1060 (1162), and $666 \mathrm{~cm}^{-1}$ are attributed to $-\mathrm{CH}_{2}$ bending vibrations, $\mathrm{O}-\mathrm{H}$ bending vibrations, $\mathrm{C}-\mathrm{O}$ stretching vibrations, and $\mathrm{C}-\mathrm{H}$ deformation vibrations of the CNFs, respectively. ${ }^{28}$ The spectra of the CNF/VGCF aerogels with different VGCF contents showed similar peaks at similar positions, indicating that the variation of VGCF content does not influence the chemical structure. As seen in the FT-IR spectra of the $\mathrm{CNF} / \mathrm{VGCF}_{1} / \mathrm{PPy}$ aerogel in Fig. 5(a), the peaks at $1794 \mathrm{~cm}^{-1}$ and $1729 \mathrm{~cm}^{-1}$ correspond to the $\mathrm{C}=\mathrm{O}$ stretching vibrations of hydroxyl groups. The peak at $1537 \mathrm{~cm}^{-1}$ is attributed to $\mathrm{C}=\mathrm{C}$ stretching vibrations of the aromatic rings in PPy. ${ }^{32}$ The peaks at 1287, 917, 1181, 1400, and $963 \mathrm{~cm}^{-1}$ are attributed to $\mathrm{C}-\mathrm{H}$ in-plane stretching vibrations, $\mathrm{C}-\mathrm{H}$ out-of-plane deformation vibrations, C-N stretching vibrations, $\mathrm{C}-\mathrm{N}$ deformation vibrations, and $\mathrm{N}-\mathrm{H}$ bending deformations of PPy, respectively. ${ }^{33,34}$ The appearance of characteristic absorption peaks of PPy indicate 
(a)

that Py was successfully in situ polymerized onto the CNF/VGCF aerogels.

Similar peaks appear in the Raman spectra of the CNF/VGCF aerogels with different VGCF contents (Fig. 4(e)). The peaks at around $1321 \mathrm{~cm}^{-1}$ and $1577 \mathrm{~cm}^{-1}$ represent the D and G bands in the VGCFs, respectively. The peak at around $2657 \mathrm{~cm}^{-1}$ is related to the 2D band of the VGCFs. ${ }^{17}$ This indicates that there was no change in the chemical structure of the aerogels with different VGCF contents, which is consistent with the FT-IR results. The ratio of $I_{\mathrm{D}}$ and $I_{\mathrm{G}}$ is $0.73(<1)$, indicating that the graphitized structure in the VGCFs suffered a small amount of destruction. Fig. 5(b) shows that the peaks of the 2D band in the $\mathrm{CNF} / \mathrm{VGCF}_{1}$ and $\mathrm{CNF} / \mathrm{VGCF}_{1} / \mathrm{PPy}$ aerogels were similar. However, the peak of the $\mathrm{D}$ band $\left(1331 \mathrm{~cm}^{-1}\right)$ shifted to $1338 \mathrm{~cm}^{-1}$, and the intensity of peak at $1577 \mathrm{~cm}^{-1}$ increased for the $\mathrm{CNF} / \mathrm{VGCF}_{1} / \mathrm{PPy}$ aerogel, indicating interactions between PPy and the VGCFs. The peaks of the characteristic groups of PPy are at $934 \mathrm{~cm}^{-1}$ and $1046 \mathrm{~cm}^{-1}$, representing ring deformation and in-plane vibrations of the $\mathrm{C}-\mathrm{H}$ deformation, respectively. ${ }^{35,36}$ It was difficult to observe the characteristic peaks of the CNFs due to the small amounts present in the composite aerogels.

XRD spectra of CNF/VGCF aerogels with different VGCF contents show that the intensities of the peaks at around $2 \theta=$ $26.5^{\circ}$ increase noticeably with increase in VGCF contents, shown in Fig. 4(f). These broad peaks with higher intensities at around $2 \theta=26.5^{\circ}$ are related to the (002) crystalline plane of graphite, showing that the VGCFs in CNF/VGCF aerogels had obvious characteristics of graphite crystals. ${ }^{21}$ Another broad diffraction peak at $2 \theta=22.5^{\circ}$ is related to (200) crystalline plane of the cellulose I structure. ${ }^{37}$ The intensities of the peak at $2 \theta=$ $22.5^{\circ}$ show no obvious change, which indicates that the addition of VGCFs had no noticeable effect on the structure of the CNFs. In addition to the similar diffraction peaks discussed above, several peaks corresponding to PPy are seen in the XRD spectra of the $\mathrm{CNF} / \mathrm{VGCF}_{1} / \mathrm{PPy}$ aerogels (Fig. 5(c)). The typical peak of amorphous PPy is at $2 \theta=26^{\circ}$, but it was not observed clearly in the XRD spectra of the $\mathrm{CNF} / \mathrm{VGCF}_{1} / \mathrm{PPy}$ aerogel because it overlaps with the peak of the (002) crystalline plane of the VGCFs. These results indicate that the addition of VGCFs did not affect the crystal structure of the CNFs and the synthesis of PPy did not affect the crystal structures of the CNFs and VGCFs.

XPS was conducted to analyze the surface elemental composition of the CNF/VFCF and CNF/VFCF/PPy aerogels. As shown in Fig. S4(b), $\uparrow$ there are three main elements, carbon, nitrogen, and oxygen, where nitrogen is present due to the PPy in the $\mathrm{CNF} / \mathrm{VFCF}_{1} / \mathrm{PPy}$ aerogel. The high-resolution XPS C $1 \mathrm{~s}$ spectra shows that there are four kinds of carbon functional groups in the $\mathrm{CNF} / \mathrm{VFCF}_{1}$ aerogel, $\mathrm{C}-\mathrm{C} / \mathrm{C}=\mathrm{C}(284.8 \mathrm{eV}), \mathrm{C}-\mathrm{O}$ (285.9 eV), $\mathrm{C}=\mathrm{O}(287.1 \mathrm{eV})$, and $\mathrm{O}-\mathrm{C}=\mathrm{O}(289.2 \mathrm{eV})$ (Fig. $5(\mathrm{~d}))^{38}$ There is an additional carbon functional group, $\mathrm{C}-\mathrm{N} / \mathrm{C}=\mathrm{N}$ $(285.5 \mathrm{eV}),{ }^{39}$ in the $\mathrm{CNF} / \mathrm{VFCF}_{1} / \mathrm{PPy}$ aerogel, along with $\mathrm{C}-\mathrm{C} / \mathrm{C}=$ $\mathrm{C}(284.8 \mathrm{eV}), \mathrm{C}-\mathrm{O}(286.3 \mathrm{eV}), \mathrm{C}=\mathrm{O}(287.1 \mathrm{eV})$ and $\mathrm{O}-\mathrm{C}=\mathrm{O}(288.6$ eV) (Fig. 5(e))..$^{15}$ Fig. $5(\mathrm{f})$ shows the high-resolution XPS N 1s spectrum of the $\mathrm{CNF} / \mathrm{VFCF}_{1} / \mathrm{PPy}$ aerogel. The peaks corresponding to $-\mathrm{NH}^{-},-\mathrm{NH}^{+}$, and $=\mathrm{NH}^{+}$in the $\mathrm{CNF} / \mathrm{VFCF}_{1} / \mathrm{PPy}$ aerogel appear at $399.6 \mathrm{eV}, 400.1 \mathrm{eV}$ and $401.2 \mathrm{eV}$, respectively. ${ }^{10}$

From the above results, it is concluded that the addition of VGCFs had no impact on the chemical structures of the 
composite aerogels and adequate amounts of PPy successfully adhered to the fibers of the CNF/VGCF aerogels through in situ polymerizing of Py monomers.

To study the electrochemical characteristics of the $\mathrm{CNF} /$ VGCF/PPy aerogel films, three-electrode tests were conducted in a $1 \mathrm{M} \mathrm{H}_{2} \mathrm{SO}_{4}$ electrolyte. The cyclic voltammetry (CV) curves were measured in a potential range of 0 to $0.8 \mathrm{~V}$. Fig. $6(\mathrm{a})$ shows the $\mathrm{CV}$ curves of the $\mathrm{CNF} / \mathrm{VGCF} / \mathrm{PPy}$ aerogel films at a scan rate of $200 \mathrm{mV} \mathrm{s}^{-1}$, both showing similar rectangular shapes, representing good charge and discharge characteristics. The size of the region enclosed by the curves increases in the order $\mathrm{VGCF}_{1}$, $\mathrm{VGCF}_{0.5}, \mathrm{VGCF}_{2}, \mathrm{VGCF}_{0.2}$, which is consistent with the results of the specific area capacitance curves. The specific area capacitances of the CNF/VGCF/PPy aerogel films do not have a positive correlation with VGCF content. This is because the structures become the prominent factor for the electrochemical properties, affecting the amount of electrolyte absorption when the electrodes are immersed in electrolyte during three-electrode testing. Therefore, even though the $\mathrm{CNF}_{\mathrm{VGCF}} / \mathrm{PPy}$ aerogel film does not possess the highest conductivity, it still performs the best in the electrochemical characterization. This is because the $\mathrm{CNF} / \mathrm{VGCF}_{1}$ aerogel has a well-formed porous network structure and has the highest specific surface area. When the content of the VGCFs is excessive, the porous structures of the hybrid aerogels are affected, reducing the absorption of electrolyte. However, when the content of the VGCFs is insufficient, the conductivity of hybrid aerogel is greatly reduced, affecting the electrode and charge transfer to some extent. However, the hydrophilicity of VGCFs is weaker than CNFs. Therefore, it is critical to select a balanced ratio of CNFs and VGCFs to form a good porous structure with a relatively high conductivity. Fig. 6(b) shows the $\mathrm{CV}$ curves of $\mathrm{CNF} / \mathrm{VGCF}_{1} / \mathrm{PPy}$ aerogel films at different scan rates of 5, 10, 50, 100 and $200 \mathrm{mV} \mathrm{s}^{-1}$. The CV curves have quasi-rectangular closed shapes with obvious redox peaks, representing reversible redox reactions caused by PPy. These can also be observed from the curves for different contents of CNF/VGCF/PPy aerogel film electrodes in Fig. S5(a)(c). $\dagger$ As shown in Fig. 6(c), the specific area capacitance of the CNF/VGCF/PPy aerogel film electrodes decreases with increase in scan rate. This is because there is sufficient time for electron transfer and diffusion to obtain higher capacitances at low scan rates. Consequently, at the scan rate of $1 \mathrm{mV} \mathrm{s}^{-1}$, the $\mathrm{CNF} /$ $\mathrm{VGCF}_{1} / \mathrm{PPy}$ aerogel film electrode obtains a maximum specific area capacitance of $5.6 \mathrm{~F} \mathrm{~cm}^{-2}$. This can be further proven by the EIS curves shown in Fig. S7(a). $\dagger$ When the curve of Nyquist plot is closer to imaginary axis in the low and medium frequency regions, the electrode is proven to have good ion diffusion. ${ }^{40}$ In other words, the greater slope of the Nyquist plot indicates a good ion diffusion process and thus good capacitance performance. Therefore, the $\mathrm{CNF} / \mathrm{VGCF}_{1} / \mathrm{PPy}$ aerogel film electrode shows the highest specific area capacitance.

Fig. 6(d), (e), S5(d) and (e) $\dagger$ show the galvanostatic chargedischarge (GCD) curves and specific gravimetric capacitances of the CNF/VGCF/PPy aerogels with different VGCF contents. These GCD curves show relatively symmetric quasi-triangular shapes. However, the discharge curves are not quite straight lines, showing obvious arc turning at around $0.5 \mathrm{~V}$. The deformation of the GCD curves is ascribed to the redox reaction
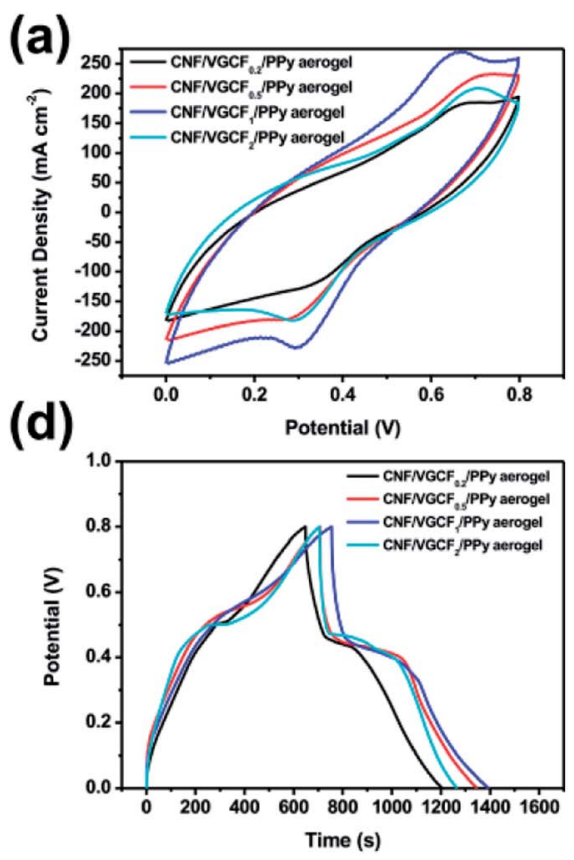

(b)
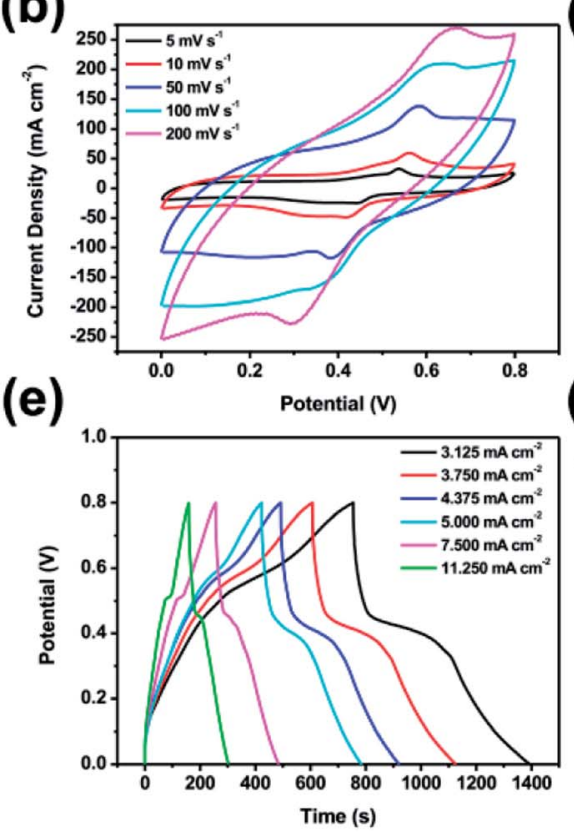
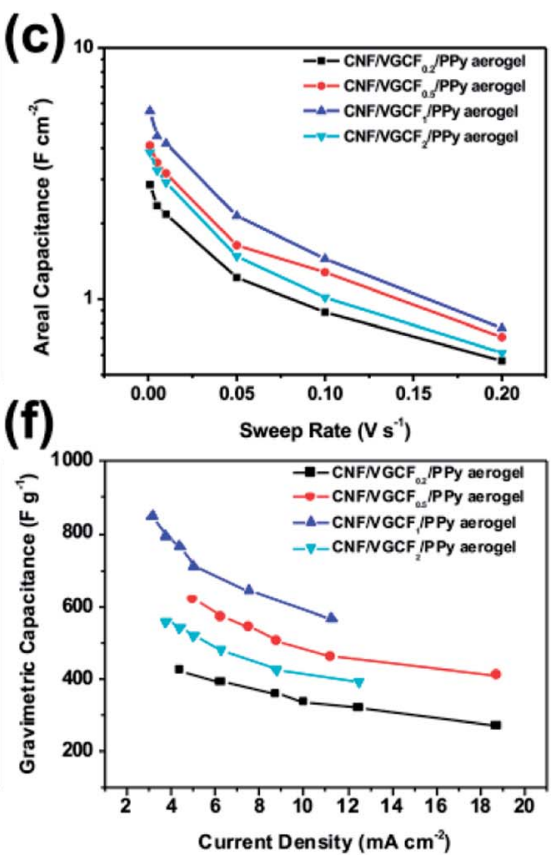

Fig. $6 \mathrm{CV}$ curves of CNF/VGCF/PPy aerogel electrodes: (a) the aerogel electrodes with different VGCF contents at $200 \mathrm{mV} \mathrm{s}{ }^{-1}$ and (b) CNF/ $V_{G C F} / P P y$ aerogel electrode at different scan rates. GCD curves of CNF/VGCF/PPy aerogel electrodes: (d) the aerogel electrodes with different VGCF contents at a current density of $3.125 \mathrm{~mA} \mathrm{~cm}{ }^{-2}$ and (e) CNF/VGCF $/$ /PPy aerogel electrode at different current densities. (c) Specific area capacitances and (f) specific gravimetric capacitances of CNF/VGCF/PPy aerogel electrodes with different VGCF contents. All data were measured in a three-electrode test. 
caused by the conductive polymer. There are small IR drops in the GCD curves related to the low interior resistance of the $\mathrm{CNF} /$ VGCF/PPy aerogel film electrodes. Theoretically, the CNF/VGCF/ PPy aerogel film electrode with a higher VGCF content should have a lower interior resistance, ${ }^{\mathbf{4 1}}$ which is also manifested by the small equivalent series resistance (ESR) from the EIS curves for the CNF/VGCF/PPy aerogel film electrodes with different VGCF contents shown in Fig. S7(a). $\dagger$ The calculated values of the ESR are $4.15,3.31,1.40$, and $0.84 \Omega$, respectively, which are relative small and decrease with increase in VGCF content. It is shown in Fig. 6(e) that the discharge time increases with decrease in current density, which can also be seen in the GCD curves shown in Fig. S5(d)-(f) $\dagger$ for different VGCF contents. There is a positive correlation between the specific gravimetric capacitance and discharge time in the GCD curves. Thus, the specific gravimetric capacitances increase with decrease in current density, as seen in Fig. 6(f). The average mass loadings of PPy for the $\mathrm{CNF} / \mathrm{VGCF}_{0.2} / \mathrm{PPy}, \mathrm{CNF} / \mathrm{VGCF}_{0.5} / \mathrm{PPy}, \mathrm{CNF} / \mathrm{VGCF}_{1} /$ PPy and $\mathrm{CNF} / \mathrm{VGCF}_{2} / \mathrm{PPy}$ aerogel films are 1.46, 1.55, 1.61, and $1.42 \mathrm{mg} \mathrm{cm}^{-2}$, respectively (Fig. $\mathrm{S} 4(\mathrm{c}) \dagger$ ). It is expected that the electrode with PPy mass loading will have a higher specific capacitance. But this could not be confirmed for the specific gravimetric capacitances and PPy mass loading in threeelectrode test. On one hand, this is because the structures of the electrodes play an important role in absorbing electrolyte, which further affects the specific capacitance. Besides, VGCFs also show the double-layer capacitance. On the other hand, the aerogel electrodes immersed in $\mathrm{H}_{2} \mathrm{SO}_{4}$ electrolyte suffer mass loss during testing, which becomes more serious with increase in the test time. For the above reasons, the maximum specific gravimetric capacitance was obtained by the $\mathrm{CNF} / \mathrm{VGCF}_{1} / \mathrm{PPy}$ aerogel film electrode with a value of $850.27 \mathrm{~F} \mathrm{~g}^{-1}$ at a current density of $3.125 \mathrm{~mA} \mathrm{~cm}{ }^{-2}$, which is higher than those of graphene/PPy-based electrodes, such as reduced graphene oxide/PPy electrode (175 $\mathrm{F} \mathrm{g}^{-1}$ ), graphene/PPy composite (354 F $\left.\mathrm{g}^{-1}\right)$ and graphene oxide/PPy/Ag electrode $\left(370.6 \mathrm{~F} \mathrm{~g}^{-1}\right) .{ }^{\mathbf{4 2} 44}$ The $\mathrm{CNF} / \mathrm{VGCF}_{0.2} / \mathrm{PPy}$ aerogel film electrode had the minimum specific gravimetric capacitance of $423.47 \mathrm{~F} \mathrm{~g}^{-1}$ at a current density of $4.375 \mathrm{~mA} \mathrm{~cm}{ }^{-2}$, which is still higher than $\mathrm{CNF} /$ PEDOT:PSS/PPy nanopaper $\left(315.5 \mathrm{~F} \mathrm{~g}^{-1}\right) .{ }^{29}$

To further study the electrochemical performance, $\mathrm{CNF} /$ VGCF/PPy aerogel films with different VGCF contents were fabricated into all-solid-state supercapacitors employing PVA/ $\mathrm{H}_{3} \mathrm{PO}_{4}$ gel as the separator and electrolyte. The electrochemical performances of the all-solid-supercapacitors were measured by a two-electrode test in a potential range of 0 to $0.8 \mathrm{~V}$. Fig. 7(a), (b) and S6(a)-(c) $\dagger$ show CV curves and specific area capacitances of the all-solid-state supercapacitors fabricated using the $\mathrm{CNF}$ / VGCF/PPy aerogel film electrodes. The CV curves are quasirectangular without obvious redox peaks. When the VGCF content is higher, the area of CV curves becomes larger, shown in Fig. 7(a), indicating higher capacitance. Fig. 7(b) shows that the shapes of $\mathrm{CV}$ curves become slender as the scan rate increases, representing lower specific area capacitances of the electrodes, which can be also observed from the CV curves in Fig. S6(a)-(c).† The calculated specific area capacitances of the CNF/VGCF/PPy aerogel film electrodes increase from 2.83 to
$8.61 \mathrm{~F} \mathrm{~cm}^{-2}$ at a scan rate of $1 \mathrm{mV} \mathrm{s}^{-1}$ with increase in VGCF content. Thus, the specific area capacitance of the $\mathrm{CNF} / \mathrm{VGCF}_{2} /$ PPy aerogel electrode had a maximum of $8.61 \mathrm{~F} \mathrm{~cm}^{-2}$ at the scan rate of $1 \mathrm{mV} \mathrm{s}^{-1}$, higher than nanocellulose/PPy/carbon material composites, such as $\mathrm{PPy} /$ carbon filaments/nanocellulose composite $\left(0.48 \mathrm{~F} \mathrm{~cm}^{-2}\right)$ and CNC/MWCNT/PPy aerogel $(2.1 \mathrm{~F}$ $\left.\mathrm{cm}^{-2}\right) .{ }^{11,16}$

Unlike the results of the three-electrode test, the maximum specific area capacitance was obtained by the $\mathrm{CNF} / \mathrm{VGCF}_{2} / \mathrm{PPy}$ aerogel electrode in the two-electrode test. This is ascribed to the structure of the aerogel films, which was affected by the pressure applied when the supercapacitor devices were fabricated. The aerogel films with higher VGCF contents had better structures than the others due to the strength of the VGCFs. Therefore, such aerogel films could absorb more electrolyte, which was beneficial for the electrochemical properties. In addition, the conductivity become an essential factor that affected the electrochemical properties when the microstructure of CNF/VGCF/PPy aerogel film electrode was affected by the second pressing. The values of the slope in the EIS curves reflect the process of ion diffusion. As shown in Fig. S7(b), $\uparrow$ a higher content of VGCF corresponds to a larger value of the slope of the $\mathrm{CNF} / \mathrm{VGCF} / \mathrm{PPy}$ aerogel film electrodes. It has also been reported that electrolyte ion diffusion is related to electronic conductivity. ${ }^{45}$ Thus, electrodes with higher VGCF contents have much better ion diffusion processes. This is an alternative explanation for why the $\mathrm{CNF} / \mathrm{VGCF}_{2} / \mathrm{PPy}$ aerogel film electrode showed better electrochemical performance.

Fig. 7(d) shows that the discharge time increases with increased VGCF content, indicating a higher capacitance of the CNF/VGCF/PPy aerogel-based supercapacitors. This is largely due to the higher conductivity of the $\mathrm{CNF} / \mathrm{VGCF} / \mathrm{PPy}$ containing higher VGCF contents. The GCD curves of the CNF/VGCF/PPy aerogel-based supercapacitors are shown in Fig. 7(e) and S6(d)-(f). $\dagger$ The shapes of the GCD curves are quasi-triangular with relatively straight lines. All the electrodes showed relatively small IR drops, indicating low internal resistances, which are beneficial for charge transport. This can be proven by the results of the EIS curves in Fig. S7(b). $\dagger$ The values of the ESR are $5.28,3.63,1.75$ and $1.11 \Omega$, respectively, decreasing with increase in VGCF content. This shows that the CNF/VGCF/PPy aerogel film electrode with a higher VGCF content and better conductivity had lower internal resistance and higher specific capacitance. In addition, the specific gravimetric capacitances of the CNF/VGCF/PPy aerogel-based supercapacitors also increased with increase in VGCF content but decreased with increase in current density, shown in Fig. $7(\mathrm{f})$. Thus, when the ratio of CNFs to VGCFs was $1 / 2$, the maximum specific gravimetric capacitances of the $\mathrm{CNF} / \mathrm{VGCF}_{2} / \mathrm{PPy}$ aerogel electrode was $678.66 \mathrm{~F} \mathrm{~g}^{-1}$ at a current density of $1.875 \mathrm{~mA} \mathrm{~cm}^{-2}$, which is higher than that of the CNF/PPy aerogel $\left(215 \mathrm{~F} \mathrm{~g}^{-1}\right),{ }^{10} \mathrm{CNF} /$ reduced graphene oxide hybrid aerogel film $\left(203 \mathrm{~F} \mathrm{~g}^{-1}\right)$, and BC/PPy/MWCNT electrode. ${ }^{32}$

The Ragone plot in Fig. 8(a) shows that both the power density and energy density increase with increase in VGCF content, from $2.79 \mathrm{~W} \mathrm{~h} \mathrm{~kg}^{-1}$ and $35.95 \mathrm{~W} \mathrm{~kg}^{-1}$ to $15.08 \mathrm{~W} \mathrm{~h} \mathrm{~kg}^{-1}$ and $334.57 \mathrm{~mW} \mathrm{~kg}^{-1}$, respectively. When the ratio of CNFs to 

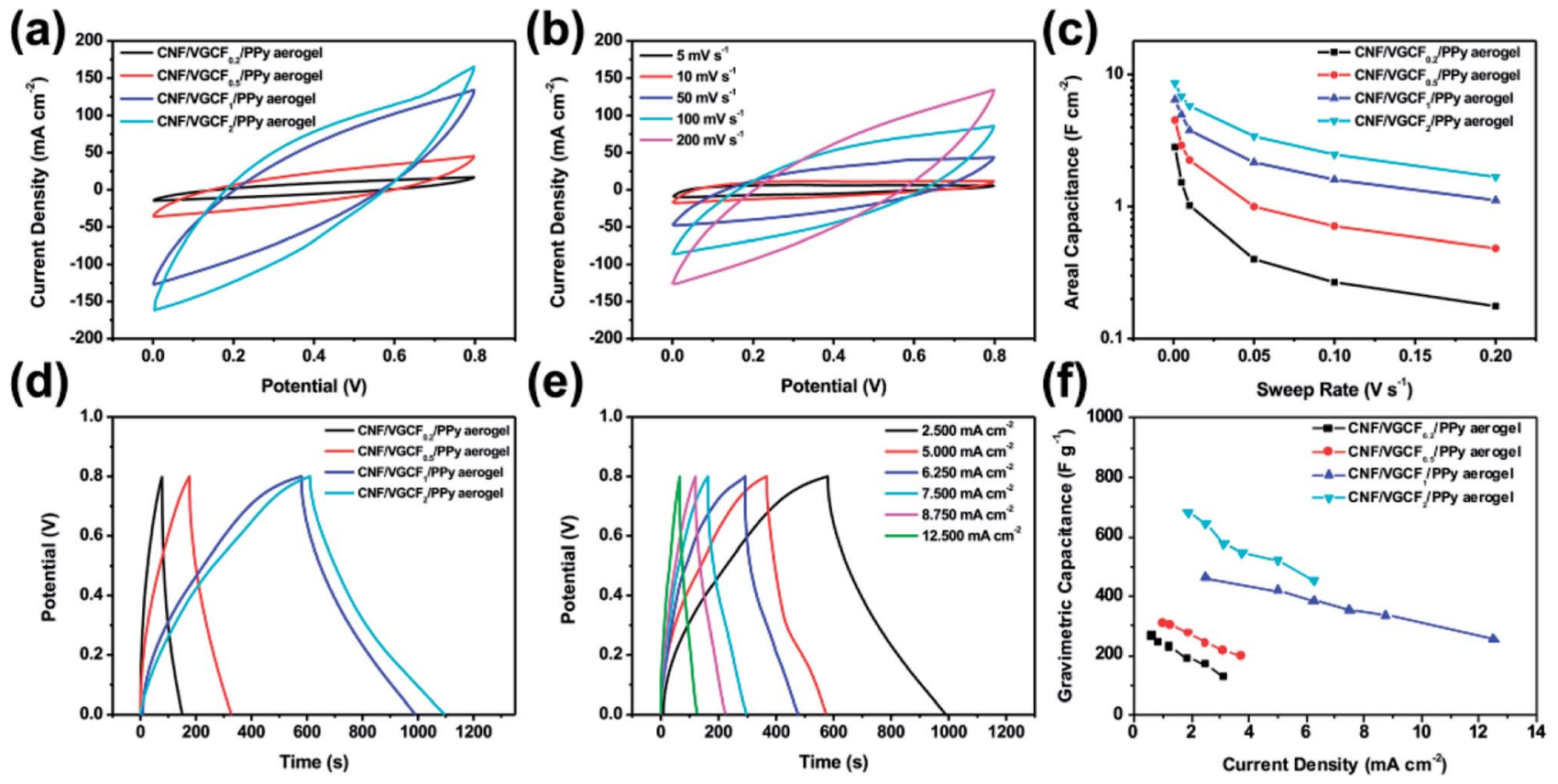

Fig. 7 CV curves of CNF/VGCF/PPy aerogel electrodes: (a) the aerogel electrodes with different VGCF content at a scan rate of $200 \mathrm{mV} \mathrm{s}^{-1}$ and (b) CNF/VGCF $/$ /PPy aerogel electrode at different scan rates. GCD curves of CNF/VGCF/PPy aerogel electrodes: (d) the aerogel electrodes with different VGCF contents at a current density of $2.500 \mathrm{~mA} \mathrm{~cm}{ }^{-2}$ and (e) CNF/VGCF $/$ /PPy aerogel electrode at different current densities. (c) Specific area capacitances and (f) specific gravimetric capacitances of CNF/VGCF/PPy aerogel electrodes with different VGCF contents. All data were measured in a two-electrode test.

VGCFs is $1 / 2$, there are maximum energy densities of $15.08 \mathrm{~W} \mathrm{~h} \mathrm{~kg}^{-1}$. When the ratio of CNFs to VGCFs is $1 / 1$, there are maximum power densities of $334.57 \mathrm{~W} \mathrm{~kg}^{-1}$.

Fig. 8(b) and S8† show the cycle stabilities of the CNF/VGCF/ PPy aerogel film electrodes with different VGCF contents after 2000 cycles at $20 \mathrm{mV} \mathrm{s}^{-1}$. The curves drop obviously from 500 to 1500 cycle and then level off in the last 500 cycles. It is likely that the sharp drop results from degradation of PPy caused by continuous swelling and shrinking during the doping and dedoping processes. ${ }^{44-47}$ The CNF/VGCF/PPy aerogel film electrodes with higher contents of VGCF had better cycle stabilities, and the $\mathrm{CNF} / \mathrm{VGCF}_{2} / \mathrm{PPy}$ aerogel film electrode retained $91.38 \%$ of its initial capacitance after 2000 cycles. This is because VGCF as the carbon nanomaterial has better cycle stability than conductive polymers. ${ }^{15}$

In summary, CNF/VGCF/PPy aerogel-based electrodes exhibited higher specific capacitances and power densities, which can be ascribed to the following characteristics of the raw materials and structural characteristics of the composite aerogel. First, the CNF/VGCF hybrid aerogels had a continuous porous network. Furthermore, the strength of the VGCFs made the structure more stable. Certainly, this is based on the premise of good dispersion of VGCFs in the CNF solution. Second, the porous structure of the aerogel and hollow structure of VGCFs provided the electrodes with a larger nano-reservoir. In addition, the electrodes could absorb more electrolyte due to the excellent hydrophilicity of the CNFs. Third, the

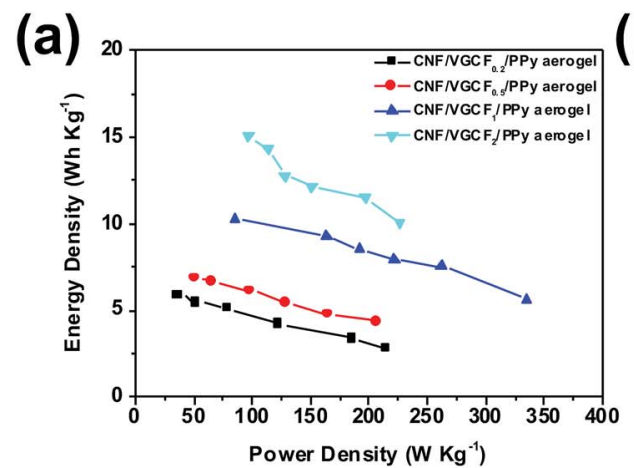

(b)

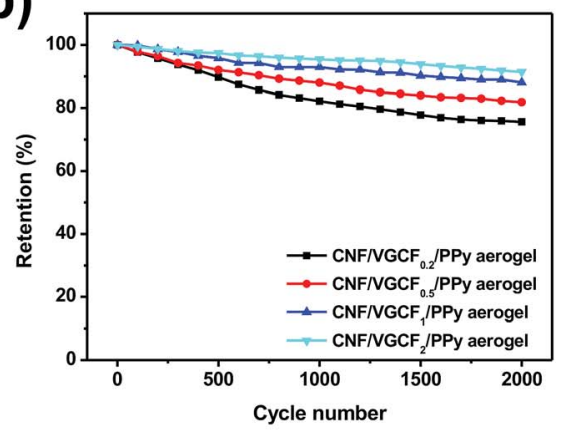

Fig. 8 (a) Ragone plot of the power density versus the energy density for CNF/VGCF/PPy aerogel electrode assembled supercapacitors. (b) Cycling stabilities of the assembled supercapacitors. 
conductivity of the electrodes was greatly improved by adding VGCFs, which promoted electron and charge transfer. Thus, due to the synergistic effects of the VGCFs and PPy, the CNF/ VGCF/PPy aerogel film electrodes obtain higher capacitances and good cycle stabilities.

\section{Conclusions}

In conclusion, the electrode materials were prepared by in situ polymerization of PPy on CNF/VGCF aerogel substrates with VGCFs forming conductive networks. The results indicated that the conductivities of the CNF/VGCF/PPy aerogel films were improved. Therefore, the $\mathrm{CNF} / \mathrm{VGCF} / \mathrm{PPy}$ aerogel electrodes showed higher capacitances of $8.61 \mathrm{~F} \mathrm{~cm}^{-2}$ at $1 \mathrm{mV} \mathrm{s}^{-1}$ (specific area capacitance) and $678.66 \mathrm{~F} \mathrm{~g}^{-1}$ at $1.875 \mathrm{~mA} \mathrm{~cm}^{-2}$ (specific gravimetric capacitance). In addition, the capacitance still retained $91.38 \%$ of its initial capacitance after 2000 cycles at $20 \mathrm{mV} \mathrm{s}^{-1}$. The superior electrochemical performances further verified that the electrode materials we prepared have potential for use in light-weight portable and wearable supercapacitors and other electric devices.

\section{Conflicts of interest}

There are no conflicts to declare.

\section{Acknowledgements}

The financial support for this work came from Special Fund for Forest Scientific Research in the Public Welfare (No. 201504603) and the Fundamental Research Funds of CAF (No. CAFYBB2017ZX003).

\section{References}

1 B. Huang, Q. Lu and L. Tang, Journal of Forestry Engineering, 2016, 1, 1-9.

2 Y. J. Kang, S. J. Chun, S. S. Lee, B. Y. Kim, J. H. Kim, H. Chung, S. Y. Lee and W. Kim, ACS Nano, 2012, 6, 64006406.

3 K. Gao, Z. Shao, X. Wang, Y. Zhang, W. Wang and F. Wang, RSC Adv., 2013, 3, 15058-15064.

4 G. Nyström, A. Marais, E. Karabulut, L. Wågberg, Y. Cui and M. M. Hamedi, Nat. Commun., 2015, 6, 7259.

5 M. Lay, I. González, J. A. Tarrés, N. Pellicer, K. N. Bun and F. Vilaseca, Eur. Polym. J., 2017, 91, 1-9.

6 Q. Yang, J. Yang, Z. Shi, S. Xiang and C. Xiong, Journal of Forestry Engineering, 2018, 3, 1-11.

7 Q. Meng, K. Cai, Y. Chen and L. Chen, Nano Energy, 2017, 36, 268-285.

8 W. Zheng, R. Lv, B. Na, H. Liu, T. Jin and D. Yuan, J. Mater. Chem. A, 2017, 5, 12969-12976.

9 X. Yang, K. Shi, I. Zhitomirsky and E. D. Cranston, Adv. Mater., 2015, 27, 6104-6109.

10 Q. Y. Niu, Y. Q. Guo, K. Z. Gao and Z. Q. Shao, RSC Adv., 2016, 6, 109143-109149.
11 K. Shi, X. Yang, E. D. Cranston and I. Zhitomirsky, Adv. Funct. Mater., 2016, 26, 6437-6445.

12 K. Devarayan, J. Park, H.-Y. Kim and B.-S. Kim, Carbohydr. Polym., 2017, 163, 153-161.

13 X. Zhang, Z. Lin, B. Chen, W. Zhang, S. Sharma, W. Gu and Y. Deng, J. Power Sources, 2014, 246, 283-289.

14 J. Tian, D. Peng, X. Wu, W. Li, H. Deng and S. Liu, Carbohydr. Polym., 2017, 156, 19-25.

15 S. Lyu, H. Chang, F. Fu, L. Hu, J. Huang and S. Wang, J. Power Sources, 2016, 327, 438-446.

16 A. Razaq, L. Nyholm, M. Sjödin, M. Strømme and A. Mihranyan, Adv. Energy Mater., 2012, 2, 445-454.

17 Z. Tang, D. Sun, D. Yang, B. Guo, L. Zhang and D. Jia, Compos. Sci. Technol., 2013, 75, 15-21.

18 R. Yuge, N. Tamura, T. Manako, K. Nakano and K. Nakahara, J. Power Sources, 2014, 266, 471-474.

19 Y. Yang, J. Xiong, J. Zeng, J. Huang and J. Zhao, Chem. Commun., 2018, 54, 1178-1181.

20 H. Tao, L. Sun, J. Li and J. Li, Acta Sci. Nat. Univ. Pekin., 2016, 52, 1155-1160.

21 F. Ma, A. Yuan and J. Xu, ACS Appl. Mater. Interfaces, 2014, 6, 18129-18138.

22 H. Liang, C. Xia, Q. Jiang, A. N. Gandi, U. Schwingenschlögl and H. N. Alshareef, Nano Energy, 2017, 35, 331-340.

23 A. Laheäär, P. Przygocki, Q. Abbas and F. Béguin, Electrochem. Commun., 2015, 60, 21-25.

24 X. Wang, Y. Zhang, H. Jiang, Y. Song, Z. Zhou and H. Zhao, Journal of Forestry Engineering, 2017, 2, 103-107.

25 H. Zhao, Y. Zhang, Y. Wang and Y. Song, Journal of Forestry Engineering, 2017, 2, 109-114.

26 L. Guo, Z. Chen, S. Lyu, F. Fu and S. Wang, Carbohydr. Polym., 2018, 179, 333-340.

27 S. Liu, K. He, X. Wu, X. Luo and B. Li, RSC Adv., 2015, 5, 87266-87276.

28 M. Lay, J. A. Méndez, M. Delgado-Aguilar, K. N. Bun and F. Vilaseca, Carbohydr. Polym., 2016, 152, 361-369.

29 M. Lay, M. À. Pèlach, N. Pellicer, J. A. Tarrés, K. N. Bun and F. Vilaseca, Carbohydr. Polym., 2017, 165, 86-95.

30 H. Y. Choi, T.-W. Lee, S.-E. Lee, J. Lim and Y. G. Jeong, Compos. Sci. Technol., 2017, 150, 45-53.

31 B. Bideau, L. Cherpozat, E. Loranger and C. Daneault, Ind. Crops Prod., 2016, 93, 136-141.

32 S. Li, D. Huang, J. Yang, B. Zhang, X. Zhang, G. Yang, M. Wang and Y. Shen, Nano Energy, 2014, 9, 309-317.

33 L. Yuan, B. Yao, B. Hu, K. Huo, W. Chen and J. Zhou, Energy Environ. Sci., 2013, 6, 470-476.

34 Z. Wang, P. Tammela, P. Zhang, M. Strømme and L. Nyholm, J. Mater. Chem. A, 2014, 2, 7711-7716.

35 Z. Chen, W. Liao and X. Ni, Chem. Eng. J., 2017, 327, 11981207.

36 Y. Liang, W. Weng, J. Yang, L. Liu, Y. Zhang, L. Yang, X. Luo, Y. Cheng and M. Zhu, RSC Adv., 2017, 7, 48934-48941.

37 Q. Liu, S. Jing, S. Wang, H. Zhuo, L. Zhong, X. Peng and R. Sun, J. Mater. Chem. A, 2016, 4, 13352-13362.

38 Q. Zheng, Z. Cai, Z. Ma and S. Gong, ACS Appl. Mater. Interfaces, 2015, 7, 3263. 
39 C. Yang, L. Zhang, N. Hu, Z. Yang, H. Wei and Y. Zhang, J. Power Sources, 2016, 302, 39-45.

40 S. Peng, L. Fan, C. Wei, X. Liu, H. Zhang, W. Xu and J. Xu, Carbohydr. Polym., 2017, 157, 344-352.

41 Z. Wang, P. Tammela, M. Strømme and L. Nyholm, Nanoscale, 2015, 7, 3418-3423.

42 J. Chen, Y. Wang, J. Cao, Y. Liu, Y. Zhou, J. H. Ouyang and D. Jia, ACS Appl. Mater. Interfaces, 2017, 9, 19831.

43 H. S. Kim, Y. Jung and S. Kim, J. Nanosci. Nanotechnol., 2017, 17, 5705-5708.
44 B. S. Singu and K. R. Yoon, Electrochim. Acta, 2018, 268, 304315.

45 L. Chen, L. Chen, Q. Ai, D. Li, P. Si, J. Feng, L. Zhang, Y. Li, J. Lou and L. Ci, Chem. Eng. J., 2018, 334, 184-190.

46 K. Gao, Z. Shao, J. Li, X. Wang, X. Peng, W. Wang and F. Wang, J. Mater. Chem. A, 2013, 1, 63-67.

47 L. Huang, W. Rao, L. Fan, J. Xu, Z. Bai, W. Xu and H. Bao, Polymers, 2018, 10, 135. 